FINNISCH-UGRISCHE STUDIEN V

GERT SAUER

\title{
DIE NOMINALBILDUNG IM OSTJAKISCHEN
}

AKADEMIE-VERLAG - BERLIN 

DIE NOMINALBILDUNG IM OSTJAKISCHEN 

FINNISCH-UGRISCHE STUDIEN V

\author{
GERT SAUER
}

\title{
DIE NOMINALBILDUNG IM OSTJAKISCHEN
}

AKADE MIE-VERLAG - BERLIN 
Erschienen im Akademie-Verlag GmbH, 108 Berlin, Leipziger Straße 3-4

Copyright 1967 by Akademie-Verlag GmbH

Lizenznummer: $202 \cdot 100 / 216 / 67$

Gesamtherstellung: VEB Druckerei "Thomas Müntzer", 582 Bad Langenaalza Bestellnummer: 2057/5 - ES $7 \mathrm{~K}$

$24,-$ 